\title{
Molecular detection of CD44v6 on aberrant crypt foci by confocal laser endoscopy
}

Although many new techniques have been developed in recent years, the detection of early neoplastic or preneoplastic lesions in the colon remains a challenge for endoscopists. In addition to adenoma, aberrant crypt foci (ACF) are considered to be precursors of adenoma and colon cancer. In fact, ACF are considered to be the earliest abnormal changes seen on the colonic surface that predispose to cancer development. ACF are clusters of altered glands that can be detected by microscopy, chromoendoscopy, or special filter techniques. Confocal laser endoscopy (CLE) is a novel imaging technique for endoscopic imaging that permits microscopic analysis of tissues during ongoing endoscopy $[1,2]$. With regard to the colon, various recent studies have demonstrated that CLE has the potential to analyze cellular and subcellular features of the mucosa in vivo. In addition, CLE analysis may be used to predict the presence of neoplastic and preneoplastic lesions in the colon. Furthermore, molecular imaging using a labeled heptapeptide has recently been shown to identify colonic adenomas with the help of CLE [3].

In initial studies, we demonstrate the case of a patient with ACF who presented for surveillance endoscopy. Colonoscopy and chromoendoscopy (Pentax EC-3870 CIFK; Pentax, Tokyo, Japan) were performed. Chromoendoscopy revealed ACF in the distal colon that were further confirmed by CLE ( $\bullet$ Fig. 1), highlighting the potential of CLE for in vivo detection of ACF in the colonic mucosa.

To further demonstrate the potential of CLE for molecular detection of markers of preneoplastic and neoplastic lesions, studies targeting the anti-CD44v6 molecule as a known colonic marker of such lesions were performed [4,5]. Accordingly, ACF-containing resected tissue was analyzed in subsequent studies using antiCD44v6 antibodies labeled with fluorescein isothiocyanate. The tissue was exposed to a 1:500 dilution of labeled antibody for 7 minutes followed by CLE. Endoscopic analysis using CLE revealed strongly positive tube-like glands indicating the expression of CD44v6 on the cell surface ( $\bullet$ Fig. 2), while labeled $\beta$-actin antibodies as negative controls showed no staining (not shown).

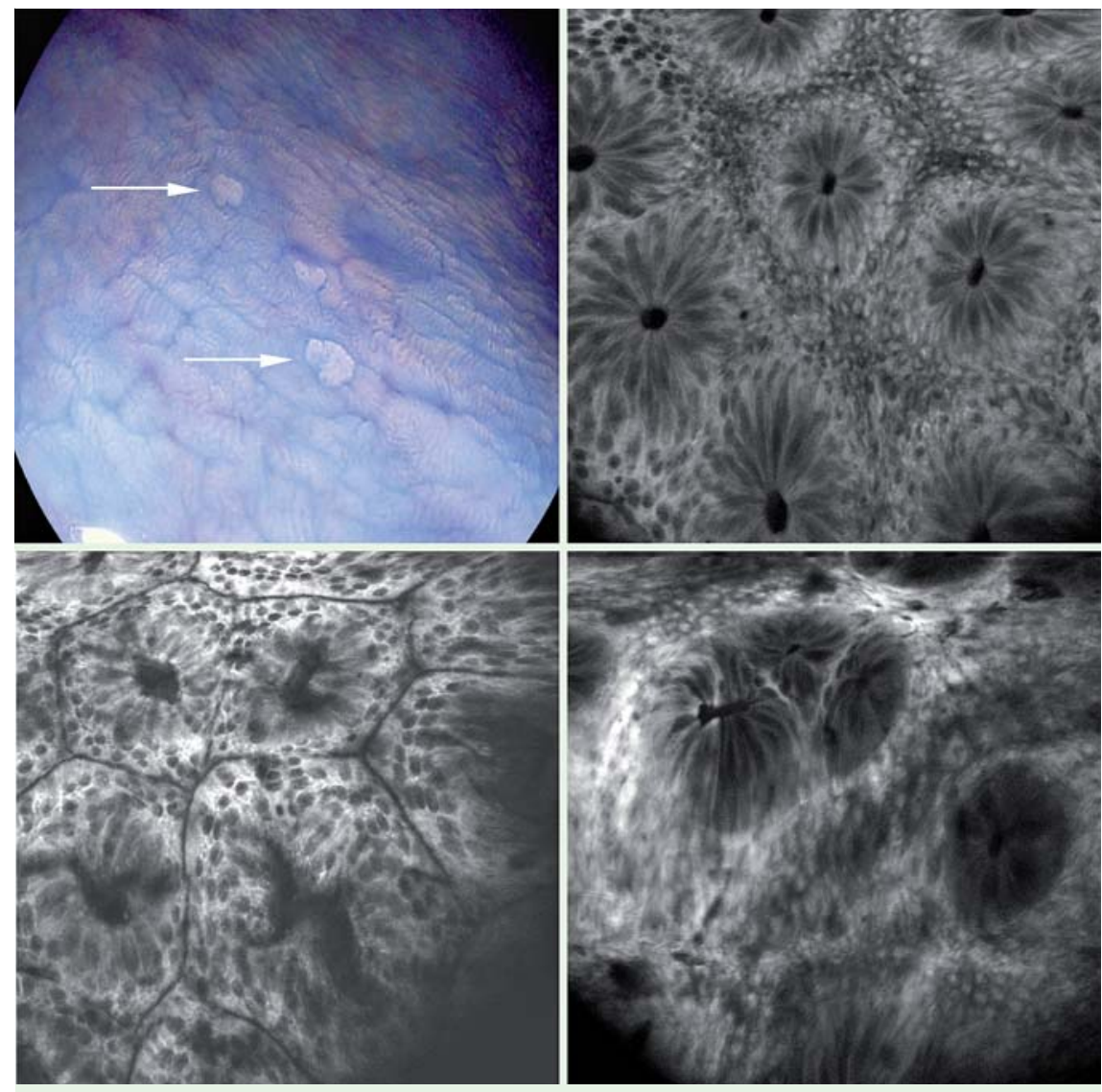

Fig. 1 Chromoendoscopy with $0.1 \%$ methylene blue highlights aberrant crypt foci (ACF) lesions in the colonic mucosa (left upper panel, arrows). In contrast to normal crypt structure (upper right panel), confocal laser endoscopy upon intravenous administration of fluorescein reveals superficial cell layers with dilated crypt openings (lower left panel) as well as clustered gland formation in deeper parts of the mucosa (lower right panel) in areas with ACF.

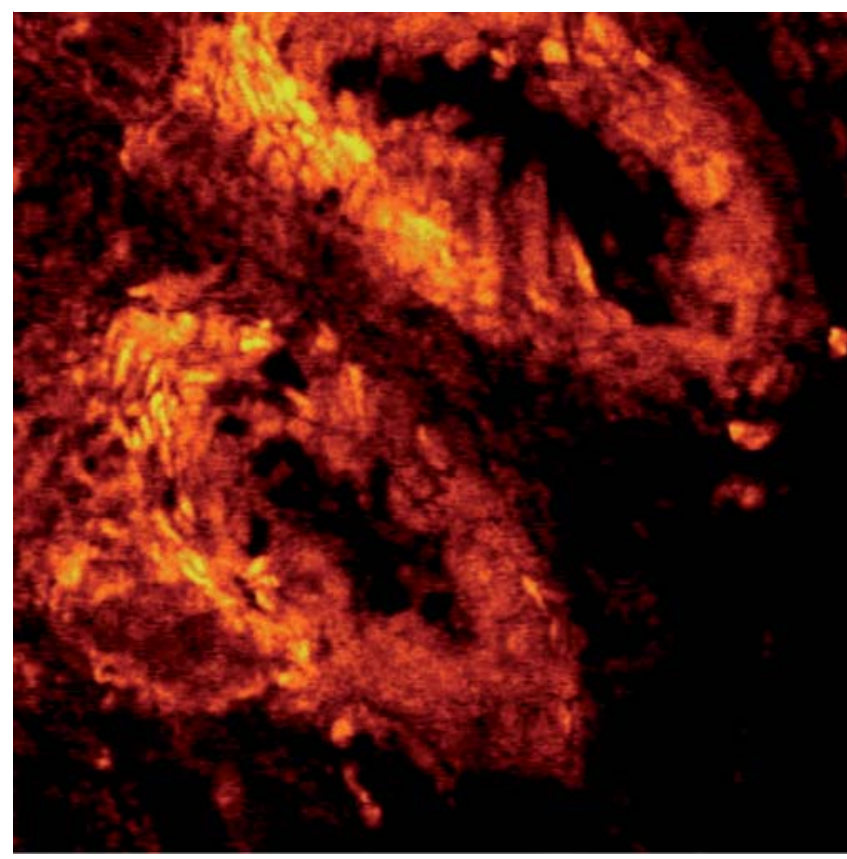

Fig. 2 Detection of CD44v6 on the surface of colonic epithelial cells. Tissue was exposed to anti-CD44v6 antibodies labeled with fluorescein isothiocyanate, followed by confocal laser endoscopy analysis. One representative confocal image is shown. 
To our knowledge, this is the first report of CLE-based molecular detection of CD44v6 expression in colonic tissue. These findings underline the potential relevance of CLE for in vivo analysis of markers and signaling molecules in colorectal diseases. Specifically, CLE was able to detect CD44v6 on colonic epithelial cells, suggesting that CLE is useful for molecular imaging. CLE with labeled molecules may thus be of relevance for early detection and functional analysis of preneoplastic and neoplastic lesions in the colon as a basis for rational endoscopic decisions and management.

Competing interests: The authors received unrestricted grants from Pentax.

Endoscopy_UCTN_Code_CCL_1AC_2AH

\section{F. Neurath ${ }^{1}$, R. Kiesslich ${ }^{2}$}

1 Department of Medicine I, University of Erlangen-Nuremberg, Germany

2 Medical Clinic I, Johannes Gutenberg University of Mainz, Germany

\section{References}

1 Kiesslich R, Goetz M, Neurath MF. Virtual histology. Best Pract Res Clin Gastroenterol 2008; 22: 883-897

2 Goetz M, Kiesslich R, Dienes HP et al. In vivo confocal laser endomicroscopy of the human liver: a novel method for assessing liver microarchitecture in real time. Endoscopy 2008; 40: 554-562

3 Hsiung PL, Hardy J, Friedland S et al. Detection of colonic dysplasia in vivo using a targeted heptapeptide and confocal microendoscopy. Nat Med 2008; 14: 454-458

4 Heider KH, Hofmann M, Horst $E$ et al. A human homologue of the rat metastasis-asso- ciated variant of CD44 is expressed in colorectal carcinomas and adenomatous polyps. J Cell Biol 1993; 120: 227-233

5 Wielenga VJ, Smits R, Korinek V et al. Expression of CD44 in Apc and Tcf mutant mice implies regulation by the WNT pathway. Am J Pathol 1999; 154: 515-523

\section{Bibliography}

DOI $10.1055 / \mathrm{s}-0030-1255812$

Endoscopy 2010; 42: E314-E315

(C) Georg Thieme Verlag KG Stuttgart · New York . ISSN 0013-726X

\section{Corresponding author}

\section{F. Neurath, MD, PhD}

Department of Medicine I

University of Erlangen-Nuremberg

Ulmenweg 18

91054 Erlangen

Germany

Fax: +49-9131-8535209

markus.neurath@uk-erlangen.de 